PART VII

MOLECULES, THEORY AND OBSERVATIONS 


\title{
SOME IMPLICATIONS OF THE DISTRIBUTION OF \\ INTERSTELLAR MOLECULES
}

\author{
LEWIS E. SNYDER, JR. \\ Astronomy Dept., University of Virginia, Charlottesville, Va. 22903, U.S.A.
}

\begin{abstract}
It is suggested that molecular observations of interstellar clouds can be used to determine the stage of cloud evolution. However, before observational data can reliably be used to investigate the evolutionary path leading from a tenuous cloud of gas and interstellar dust to a star cluster, mechanisms of molecular formation, excitation, and destruction must be taken into account. The evidence is growing that interstellar dust grains have an important role in molecular formation and destruction.
\end{abstract}

\section{Introduction}

In the past few years a number of interesting and informative review articles on interstellar molecules have been published (Buhl, 1971; Buhl and Snyder, 1971; Heiles, 1971; McNally, 1968; Rank et al., 1971; Snyder, 1972; Snyder and Buhl, 1970; Turner, 1970) and are recommended reading for background information. In addition, many of the papers presented at this meeting represent a significant contribution to the study of the interrelationship between dust and molecules. In this review, I have been asked to discuss the distribution of interstellar molecules. Since the true galactic distribution of most of the twenty-six known molecules is not yet known, I will approach this problem by suggesting tentative classifications for molecular clouds which I hope will influence those of you who are planning future molecular survey work. In particular, it is important for astronomers to begin to think of interpreting molecular survey data in terms of the evolution of dust clouds. Before this can be done meaningfully, however, it becomes necessary to correctly interpret abundances and excitation and to understand the dominant molecular formation mechanisms.

\section{Molecular Abundances}

Tables I and II list the known inorganic and organic interstellar molecules. In each table, the first column gives the chemical formula and name, the second the approximate number of known sources in our Galaxy, the third column the range of projected densities in $\mathrm{cm}^{-2}$ (number density integrated along the line-of-sight); the fourth column lists the spectral region where the molecule has been observed; and the last column indicates whether the molecular line is observed in absorption, emission, or both. A molecular source is defined as a given direction in the Galaxy which typically contains multiple molecular clouds along the line of sight. Hence the source counts listed in column two of both Tables I and II may be regarded as lower bounds on the actual number of molecular clouds observed. 
TABLE I

Inorganic interstellar molecules

\begin{tabular}{lllll}
\hline & $\begin{array}{l}\text { No. reported } \\
\text { sources }\end{array}$ & $\begin{array}{l}\text { Projected } \\
\text { density }\left(\mathrm{No} \mathrm{cm}^{-2}\right)\end{array}$ & $\begin{array}{l}\text { Spectral } \\
\text { region }\end{array}$ & $\begin{array}{l}\text { Absorption (A) } \\
\text { or emission (E) }\end{array}$ \\
\hline $\begin{array}{l}\text { Diatomic molecules } \\
\mathrm{H}_{2} \text { - molecular hydrogen }\end{array}$ & $1(\xi \mathrm{Per})$ & $\sim 10^{20}$ & Far UV & $\mathrm{A}$ \\
$\mathrm{OH}-$ hydroxyl & $>200$ & $10^{12}-10^{16}$ & $+\mathrm{R}$ & $\mathrm{A}, \mathrm{E}$ \\
$\mathrm{SiO}-$ silicon monoxide & $1(\mathrm{Sgr} \mathrm{B} 2)$ & $\sim 10^{13}$ & $\mathrm{R}$ & $\mathrm{E}$ \\
$\begin{array}{l}\text { Triatomic } \\
\mathrm{H}_{2} \mathrm{O} \text { - water }\end{array}$ & $>50$ & - & $\mathrm{R}$ & $\mathrm{E}$ \\
$\mathrm{H}_{2} \mathrm{~S}$ - hydrogen sulfide & $>9$ & $10^{13}-10^{14}$ & $\mathrm{R}$ & $\mathrm{E}$ \\
Four-atomic & & & & \\
$\mathrm{NH}_{3}$ - ammonia & $<10$ & $\sim 10^{16}$ & $\mathrm{R}$ & $\mathrm{E}$ \\
\hline$+\mathrm{R}=$ Radio Detection & & & &
\end{tabular}

${ }^{+} \mathrm{R}=$ Radio Detection

TABLE II

Organic interstellar molecules

\begin{tabular}{|c|c|c|c|c|}
\hline & $\begin{array}{l}\text { No. reported } \\
\text { sources }\end{array}$ & $\begin{array}{l}\text { Projected } \\
\text { density }\left(\mathrm{No} \mathrm{cm}^{-2}\right)\end{array}$ & $\begin{array}{l}\text { Spectral } \\
\text { region }\end{array}$ & $\begin{array}{l}\text { Absorption (A) } \\
\text { or emission (E) }\end{array}$ \\
\hline \multicolumn{5}{|l|}{ Diatomic molecules } \\
\hline $\mathrm{CH}^{+}$ & $\sim 60$ & $\sim 10^{13}$ & $+\mathrm{O}$ & A \\
\hline $\mathrm{CH}$ & 40 & $\sim 10^{13}$ & o & A \\
\hline $\mathrm{CN}$ - cyanogen radical & $\sim 14 / 3$ & $\sim 10^{12} / 10^{15}$ & $\mathbf{O} / \mathbf{R}$ & $\mathrm{A}, \mathrm{E}$ \\
\hline $\mathrm{CO}$ - carbon monoxide & Many/1 ( $\zeta \mathrm{Oph})$ & $10^{17}-10^{19} / 10^{15}$ & $\mathrm{R} / \mathrm{O}$ & E/A \\
\hline CS - carbon monosulfide & 4 & $10^{13}-10^{14}$ & $\mathbf{R}$ & $\mathrm{E}$ \\
\hline \multicolumn{5}{|l|}{ Triatomic } \\
\hline HCN - hydrogen cyanide & $\sim 10$ & $10^{14}-10^{15}$ & $\mathbf{R}$ & $\mathrm{E}$ \\
\hline OCS - carbonyl sulfide & 1 (Sgr B2) & $\sim 10^{15}$ & $\mathbf{R}$ & $\mathrm{E}$ \\
\hline \multicolumn{5}{|l|}{ Four-atomic } \\
\hline $\mathrm{H}_{2} \mathrm{CO}$ - formaldehyde & Many $(>100)$ & $10^{12}-10^{16}$ & $\mathbf{R}$ & $\begin{array}{l}\text { A }(6 \mathrm{~cm} \text { line), } \\
\text { E (all other lines) }\end{array}$ \\
\hline HNCO - isocyanic acid & 1 (Sgr B2) & $\sim 10^{14}$ & $\mathbf{R}$ & E \\
\hline $\mathrm{H}_{2} \mathrm{CS}$ - thioformaldehyde & 1 (Sgr B2) & - & $\mathbf{R}$ & A \\
\hline \multicolumn{5}{|l|}{ Five-atomic } \\
\hline $\mathrm{H}_{2} \mathrm{CNH}$ - methylenimine & 1 (Sgr B2) & - & $\mathbf{R}$ & $\mathrm{E}$ \\
\hline $\mathrm{HCOOH}$ - formic acid & 1 (Sgr B2) & $<10^{13}$ & $\mathbf{R}$ & $\mathrm{E}$ \\
\hline $\mathrm{HC}_{3} \mathrm{~N}$ - cyanoacetylene & 2 or more & $\sim 10^{16}$ & $\mathbf{R}$ & $\mathrm{E}$ \\
\hline \multicolumn{5}{|l|}{ Six-atomic } \\
\hline $\mathrm{CH}_{3} \mathrm{OH}$ - methyl alcohol & 3 or more & - & $\mathbf{R}$ & $\mathrm{E}$ \\
\hline $\mathrm{CH}_{3} \mathrm{CN}$ - methyl cyanide & & $\sim 10^{14}$ & $\mathbf{R}$ & $\mathrm{E}$ \\
\hline $\mathrm{HCONH}_{2}$ - formamide & 2 or more & $>10^{11}$ & $\mathbf{R}$ & $\mathrm{E}$ \\
\hline \multicolumn{5}{|l|}{ Seven-atomic } \\
\hline $\mathrm{CH}_{3} \mathrm{C}_{2} \mathrm{H}$ - methylacetylene & 1 (Sgr B2) & $\sim 10^{14}$ & $\mathbf{R}$ & $\mathrm{E}$ \\
\hline $\mathrm{HCOCH}_{3}$ - acetaldehyde & 2 & - & $\mathbf{R}$ & $\mathrm{E}$ \\
\hline \multicolumn{5}{|l|}{ Other } \\
\hline$X$-ogen $(\mathrm{CCH}$ ?) & 8 & - & $\mathbf{R}$ & $\mathrm{E}$ \\
\hline 'HNC' (hydrogen isocyanide) & $>5$ & - & $\mathbf{R}$ & $\mathrm{E}$ \\
\hline
\end{tabular}

${ }^{+} \mathrm{O}=$ Optical detection 
The majority of interstellar molecules have been observed in the radio region of the spectrum. Two inherent advantages are gained by using radio techniques which usually more than offset the disadvantages of small line strengths and the molecular polarity required for pure rotational transitions. The first advantage is that radio waves are capable of penetrating dense dust clouds where complex molecules presumably are afforded the maximum degree of protection against photoionization and photodissociation. Optical observers, on the other hand, must rely on a bright background star as an absorption source when searching the intervening clouds for interstellar molecules; thus such observations are necessarily limited to low opacity clouds which don't offer much protection for molecules with low photodestruction thresholds. The second advantage is the low excitation required by pure rotational transitions. The molecular excitation, $E$, may be written

$$
E=E_{\mathrm{rot}}+E_{\mathrm{vib}}+E_{\mathrm{el}}
$$

where for molecules of interstellar interest the rotational energy, $E_{\text {rot }}$, may be only $10^{2}$ $\mathrm{cm}^{-1}$ or less; compare this with typical vibrational energy $\left(E_{\mathrm{vib}}\right)$ values of $10^{3} \mathrm{~cm}^{-1}$ or more and electronic excitation energies $\left(E_{\mathrm{el}}\right)$ of $\sim 10^{4} \mathrm{~cm}^{-1}$. Hence radio transitions $\left(E_{\text {rot }}\right)$ may be excited by low energy sources such as collisions or radio radiation from $H$ II regions but infrared, optical and ultraviolet transitions require a background star with intervening cloud. Due to these physical differences in excitation mechanisms, the few molecules listed in Tables I and II which have been detected by optical or ultraviolet spectroscopy have been observed in low opacity clouds against hot $\mathrm{O}$ and B stars and tend to have simpler chemical composition than those molecules detected by radio techniques in denser dusty regions such as the Sagittarius complex or the circumstellar region associated with IRC +10216.

The projected densities listed in Tables I and II are representative samples taken from various interstellar regions. Comparison of molecular densities observed in the same region is particularly difficult for radio observations due to beam dilution and nonhomogeneous excitation across the dimension of a typical beamwidth. For example, Figure 1 illustrates typical telescope beamwidths projected on the Orion Nebula. The ideal halfpower beamwidth (HPBW) is given for a parabolic radio telescope (in minutes of arc) by

$$
\mathrm{HPBW}=4125.4 \cdot v(\mathrm{GHz})^{-1} \cdot D(\mathrm{ft})^{-1}=138.6 \cdot \lambda(\mathrm{cm}) \cdot D(\mathrm{ft})^{-1},
$$

where the observational frequency $v$ is in $\mathrm{GHz}$, the observational wavelength $\lambda$ is in $\mathrm{cm}$, and the telescope diameter $D$ is in $\mathrm{ft}$. Thus, as shown in Figure 1, an OH observation with a single element telescope at $18 \mathrm{~cm}$ wavelength covers a much broader region of the molecular source than an $\mathrm{HCN}$ observation at $3 \mathrm{~mm}$ wavelength. Hence the correct interpretation of molecular excitation can be difficult for radio molecules.

Probably it is safe to say that none of the radio molecules detected to date is in thermal equilibrium. We might think of the following three general categories of molecules, characterized by their energy level structures, which are useful for assigning relative reliabilities to reported abundances of radio molecules. 


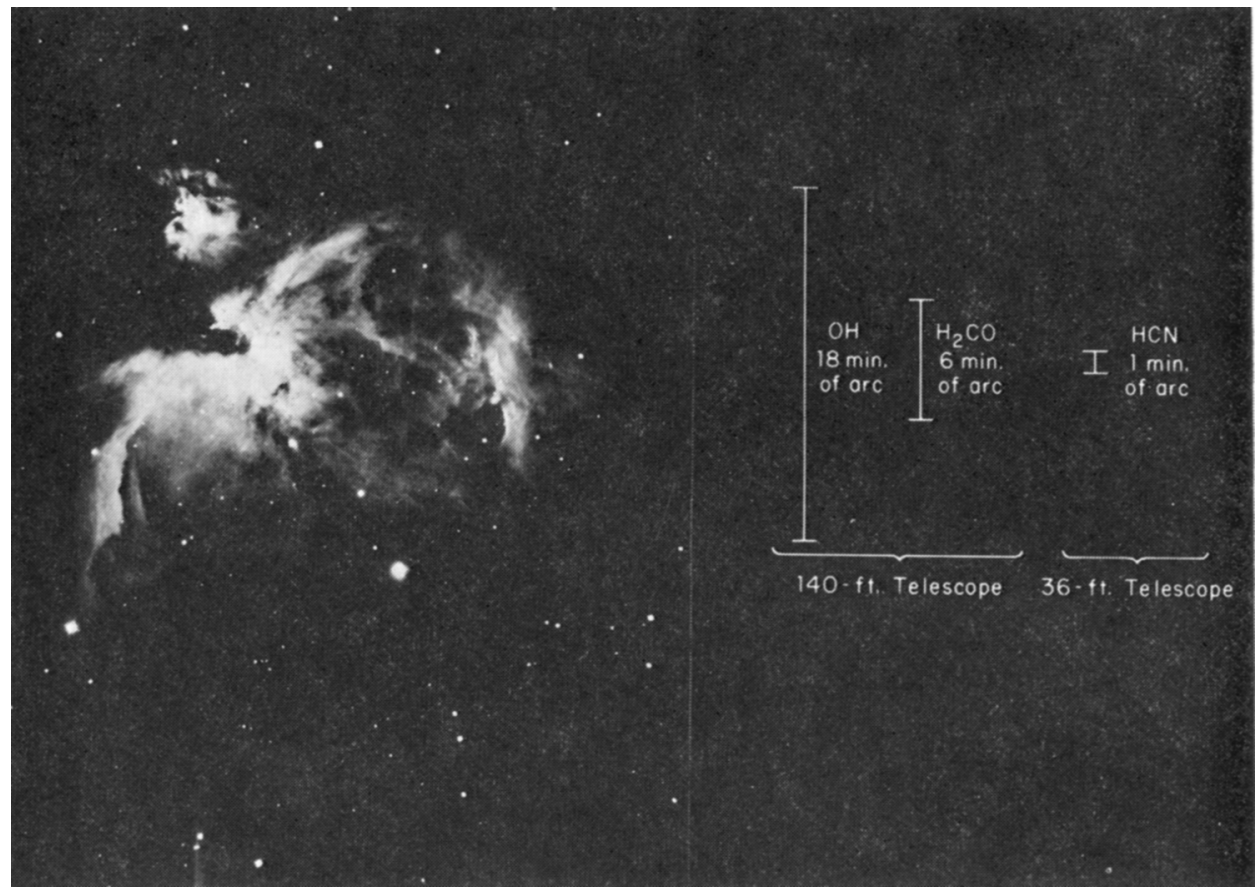

Fig. 1. The spatial resolution (HPBW) of the NRAO 140-ft telescope is illustrated against the Orion Nebula for the $18 \mathrm{~cm}$ transitions of $\mathrm{OH}$ and the $6.2 \mathrm{~cm}$ transitions of formaldehyde $\left(\mathrm{H}_{2} \mathrm{CO}\right)$. Compare the relatively large HPBW obtained for $\mathrm{OH}$ and $\mathrm{H}_{2} \mathrm{CO}$ with the small $\mathrm{HPBW}$ of the NRAO 36-ft telescope used to observe the $3.4 \mathrm{~mm}$ transition of hydrogen cyanide (HCN). Hence it is difficult to compare radio observations of the same region which are made at different wavelengths (Buhl and Snyder, 1971).

(A) Extreme Maser Action. These molecules have energy level structures with accompanying selection rules which permit both radiative and collisional pumping to occur. Typical examples are $\mathrm{H}_{2} \mathrm{O}$, an asymmetric rotator, and $\mathrm{OH}$, a diatomic molecule with lambda-type doubling. Figure 2 illustrates the fairly complex ground state energy level schemes of $\mathrm{OH}, \mathrm{H}_{2} \mathrm{O}$ and the recently discovered (Thaddeus et al., 1972) interstellar $\mathrm{H}_{2} \mathrm{~S}$.

(B) Mild Maser Action. These molecules have selection rules which allow strong radiative (electric dipole) transitions only between immediately adjacent levels in the ground electronic state. Hence radiative pumping is much more difficult but collisional pumping - which usually is much weaker - may still be allowed. These molecules are either diatomics with energy levels uncomplicated by Coriolis or quadrupole interactions (e.g. $\mathrm{CO}$ and $\mathrm{CS}$ ) or symmetric rotators such as $\mathrm{CH}_{3} \mathrm{C}_{2} \mathrm{H}$ (Figure 3) where all energy levels with a given angular momentum projection quantum number $K$ may behave exactly as a simple diatomic molecule. Due to its extremely small dipole moment $(\sim 0.1 \mathrm{D})$ and simple level structure, $\mathrm{CO}$ is probably closer to equilibrium with the surrounding gas than any other molecule detected so far. Hence if saturation 


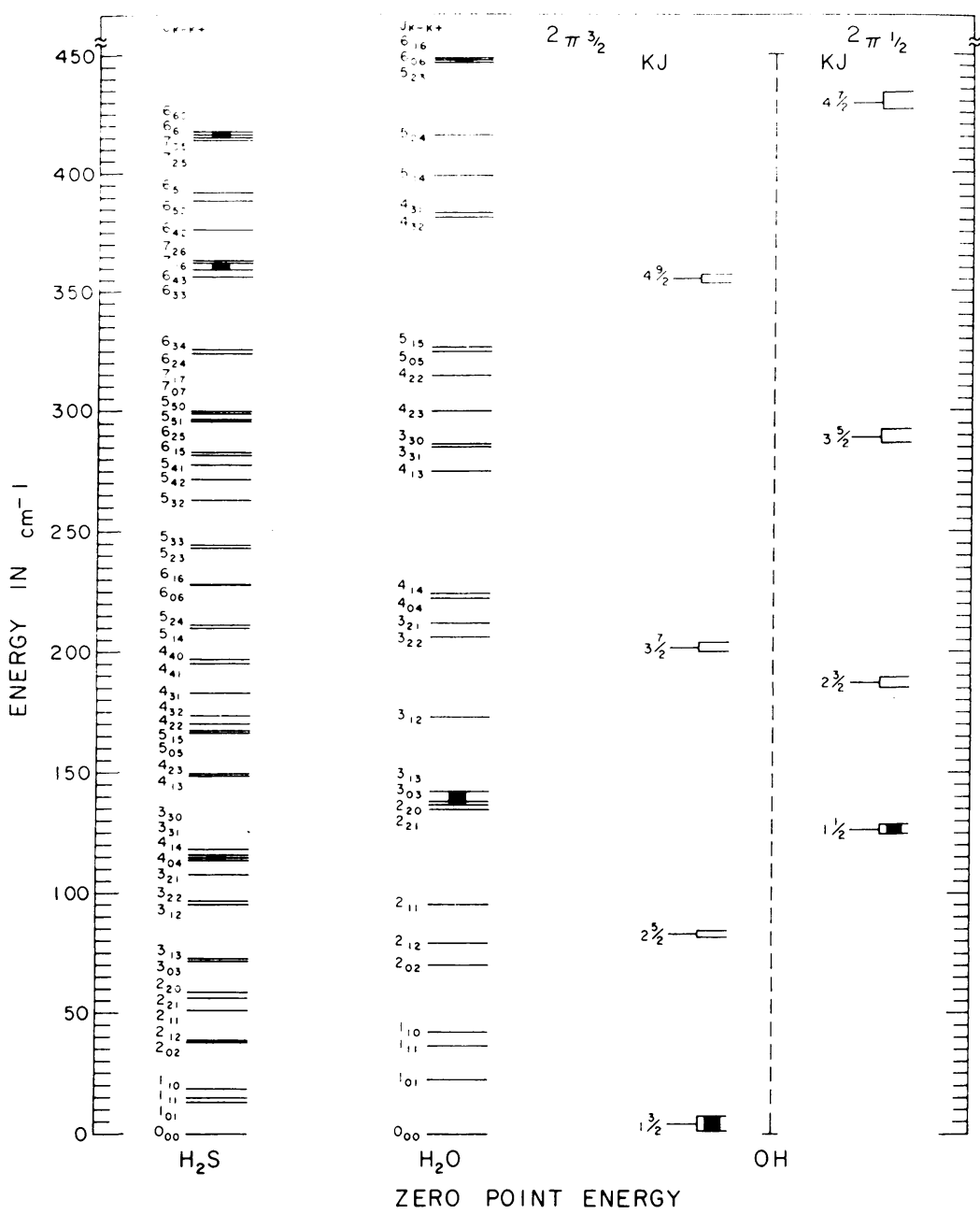

Fig. 2. The ground state rotational energy levels of several molecules which might be expected to exhibit masering are compared. Here it can be seen that the energy levels of hydrogen sulfide $\left(\mathrm{H}_{2} \mathrm{~S}\right)$ bear very little resemblance to those of water $\left(\mathrm{H}_{2} \mathrm{O}\right)$ and neither molecule has the relatively simple level structure of $\mathrm{OH}$. The bars indicate the permitted radio frequency transitions $6_{60} \rightarrow 7_{35}, 7_{16} \rightarrow$ $6_{43}$ and $3_{31} \rightarrow 4_{04}$ in $\mathrm{H}_{2} \mathrm{~S}$ (all currently undetected in the interstellar medium); $6_{16} \rightarrow 5_{23}$ (detected) and $3_{13} \rightarrow 2_{20}$ (undetected) in $\mathrm{H}_{2} \mathrm{O}$; and two of the typical lambda-doublet type transitions in $\mathrm{OH}$. The $\mathrm{OH}$ lambda-doublet hyperfine splitting is not illustrated.

effects are properly taken into account, $\mathrm{CO}$ abundances should be among the most reliable of those listed in Tables I and II.

(C) Intermediate Maser Action. This class of molecules has not yet been thoroughly studied but is possibly the most interesting of the three. Here, due to the molecular geometry, we find symmetry groups of rotational energy levels with selection rules and excitation similar to both class A and class B molecules. An example might be HNCO which has pseudo 'parastates' (Figure 4) which may have excitation similar to a simple 


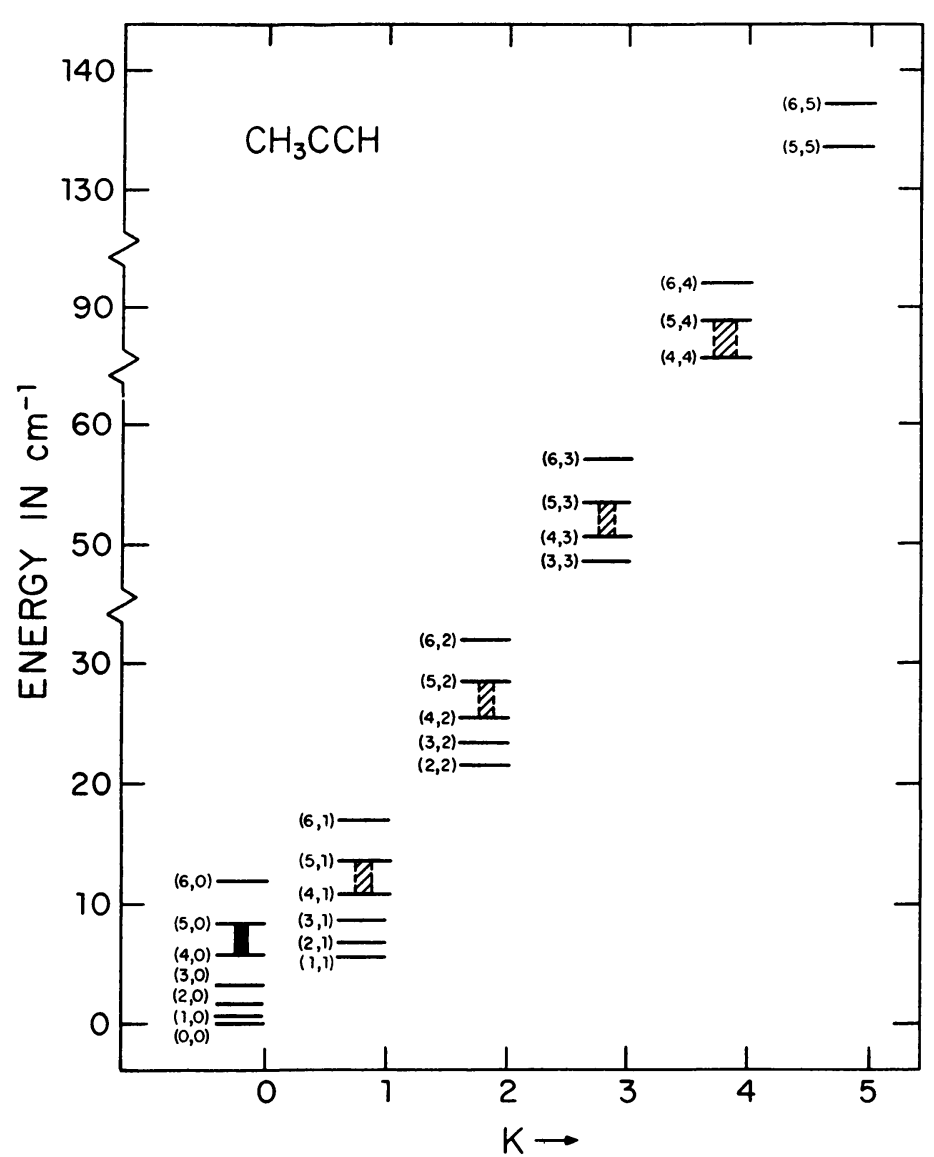

Fig. 3. The lowest ground state rotational energy levels of a fairly typical symmetric rotator, methylacetylene $\left(\mathrm{CH}_{3} \mathrm{C}_{2} \mathrm{H}\right)$, are relatively uncomplicated when compared with the analogous levels in an asymmetric rotator. Individual levels are labeled with the quantum numbers $(J, K)$ and, in the absence of strong collisional interactions, each set of levels with the same $K$ value is effectively isolated from every other set and hence resembles the level scheme for a simple diatomic molecule. The solid bar represents the most intense component of the detected interstellar line at $3.5 \mathrm{~mm}$; the dashed bars represent weaker transitions.

class B molecule while the 'orthostates' are structured similarly to $\mathrm{H}_{2} \mathrm{CO}$, a class A molecule, and hence may be strongly pumped. Other examples may be $\mathrm{HCN}$ and $\mathrm{HC}_{3} \mathrm{~N}$ which have linear structures and hence would be expected to fit into class $\mathrm{B}$. However, the nitrogen atom introduces quadrupole splitting of the rotational levels which may contribute to non-LTE population distributions when the line widths are less than the quadrupole separation.

\section{Molecular Cloud Classifications}

The molecular cloud observations of the past few years have shown that several types of molecular clouds exist which vary greatly in chemical composition, density and 


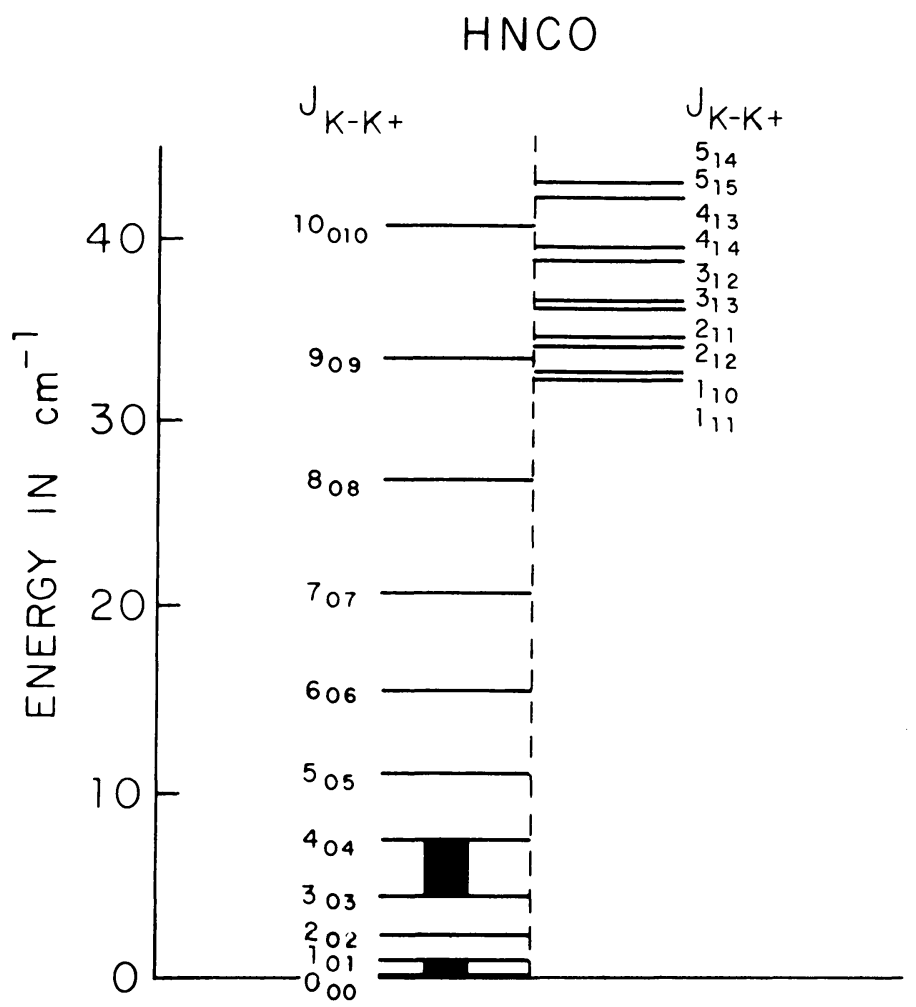

Fig. 4. The lowest ground state rotational energy levels of isocyanic acid (HNCO) are illustrated with the pseudo 'parastates' on the left-hand side and 'orthostates' on the right. Although the level structure is similar to formaldehyde $\left(\mathrm{H}_{2} \mathrm{CO}\right)$ and thioformaldehyde $\left(\mathrm{H}_{2} \mathrm{CS}\right)$, the ortho and para separation does not really exist for $\mathrm{HNCO}$ because relatively weak radiative transitions are allowed across the dotted line.

excitation. In some cases, it is possible to distinguish between prestellar and stellar regions of excitation; furthermore, we might invoke the following molecular cloud classifications:

(A) Clouds associated with $\mathrm{H}$ il regions. These molecular clouds have nearby $\mathrm{H}$ II regions and may contain infrared objects which often are believed to be recently formed. The neutral particle densities found from collisional excitation calculations indicate that these clouds are very massive $\left(10^{3}-10^{6} M_{\odot}\right)$. In addition, high temperature knots of $100 \mathrm{~K}$ or greater are occasionally found. Clouds of this type are of interest because they contain detectable abundances of the most complex molecular species in our Galaxy. Good examples are the galactic center clouds (in the direction of Sgr A and Sgr B2), the clouds associated with the Orion Nebula and the W51 region. Clouds in this category may contain both prestellar and stellar components.

(B) Isolated clouds not physically associated with excitation sources. There may be two subcategories of isolated molecular clouds; both are prestellar.

(1) The first category would include those of moderate mass $\left(<10^{3} M_{\odot}\right)$ and kinetic temperatures of 50-100 K. The detectable molecules may include species such 
as $\mathrm{CN}, \mathrm{CH}, \mathrm{CO}, \mathrm{OH}$ and/or $\mathrm{H}_{2} \mathrm{CO}$ but nothing of very complex chemical composition. Examples in this category would include the Perseus and Orion arm regions (Davies and Matthews, 1972) in the direction of Cas A and possibly the well-known $\zeta$ Oph cloud. The simple chemistry implies that the density distribution does not effectively support the formation or prevent the destruction of complex molecules.

(2) The second subcategory of isolated clouds would include dark nebulae which have 6-8 mag. of extinction and are fairly cool $(15-50 \mathrm{~K})$. Here the density distribution is probably high enough to support sophisticated interstellar chemistry but the low excitation makes detection difficult. Examples in this category would be Bok Globules and dark nebulae where $\mathrm{H}_{2} \mathrm{CO}$ is observed in absorption against the $3 \mathrm{~K}$ background.

(C) Stellar Objects. In this case, molecular observations have shown three subcategories of objects, all of which are stellar.

(1) Mira-type variables. Radio observations show time-variable $\mathrm{OH}$ and $\mathrm{H}_{2} \mathrm{O}$ signals which change in a semipredictible manner.

(2) Carbon stars with circumstellar shells. Simple but stable molecules such as $\mathrm{CO}$, $\mathrm{CN}, \mathrm{HCN}$ and CS have been detected in the direction of IRC+10216 and several other stars with infrared excess. However, the chemical complexity is not yet as interesting as that of class A objects.

(3) 'Protostars'. This is a tentative classification for the high density knots within class A clouds. In some cases, these knots may correspond to recently detected infrared sources. Typically, the signal intensity of a complex molecule may double in the direction of a density knot and localized, intense $\mathrm{H}_{2} \mathrm{O}$ and $\mathrm{OH}$ maser emission may be observed. Thus the high densities and unusual excitation suggest that regions of this type may be in the process of undergoing stellar formation.

The foregoing discussion illustrates that there is no such thing as a 'typical' molecular cloud but instead several cloud categories, each containing specific information. Possibly a solution to this problem would be a broad cloud classification scheme from which we would attempt to deduce the stage of evolution of interstellar clouds. In general, we would only be completing our picture of the stellar life cycle by trying to categorize the physical properties of dust clouds beginning sometime after stellar death and continuing to the initial stages of stellar formation. Thus we could think of a general cloud classification scheme as the other half of a very complete stellar evolutionary track in an HR diagram. An invaluable tool for this classification will be the kinds and abundances of molecules found in the clouds. In order to use interstellar molecules as a measure of cloud evolution, however, it is also critical to understand molecular formation processes.

\section{Comments on Some Suggested Formation Processes}

From the wide variety of molecules found (Tables I and II) it is probable that more than one formation mechanism is responsible for the observed species. Over the years numerous formation processes have been suggested which deserve some comment in light of molecular observations. 
(1) Vapor-Phase Reactions. Two-body radiative association and inverse predissociation reactions have been used to predict (Solomon and Klemperer, 1972, Julienne et al., 1971; Stecher and Williams, 1973) the abundances of diatomics such as $\mathrm{CH}$, $\mathrm{CH}^{+}, \mathrm{CN}, \mathrm{OH}$ and $\mathrm{CO}-$ usually for the interstellar cloud associated with $\zeta \mathrm{Oph}$. This type of calculation is important but it should be emphasized that the polyatomic molecules are not being found in the $\zeta$ Oph cloud and the difficult chemistry lies in addressing questions such as why isocyanic acid (HNCO) densities reach $10^{-5} \mathrm{~cm}^{-3}$ in the direction of Sgr B2 and why molecules such as acetaldehyde $\left(\mathrm{HCOCH}_{3}\right)$ and methylacetylene $\left(\mathrm{CH}_{3} \mathrm{C}_{2} \mathrm{H}\right)$ can be formed at all in the interstellar clouds. Current observational evidence suggests that formation mechanisms more efficient than vaporphase reactions are needed to explain the abundances of the heavy polyatomics. However, in view of the extremely high $\mathrm{H}_{2}$ densities inferred from studies of collisional excitation of microwave lines, it appears to be important to study reactions which utilize $\mathrm{H}_{2}$ to form simple polyatomics such as $\mathrm{H}_{2} \mathrm{CO}, \mathrm{H}_{2} \mathrm{O}$ and $\mathrm{NH}_{3}$. In addition, the potential role of $\mathrm{CH}_{4}$ in the formation of $\mathrm{CH}_{3} \mathrm{CN}$ and $\mathrm{CH}_{3} \mathrm{C}_{2} \mathrm{H}$ as well as the importance of $\mathrm{C}_{2} \mathrm{H}_{2}$ to the formation of $\mathrm{HC}_{3} \mathrm{~N}$ and $\mathrm{CH}_{3} \mathrm{C}_{2} \mathrm{H}$ should be studied through vapor-phase formation models.

(2) Stellar Ejection of Molecules. Although this mechanism for molecular formation has been discussed in the literature many times, at present there is no radio evidence that this process contributes significantly to the observed abundances. There are, however, radio observations of several circumstellar shells (discussed by Buhl (1971), Buhl and Snyder (1971), Heiles (1971), NcNally (1968), Rank et al. (1971), Snyder (1972), Snyder and Buhl (1970), and Turner (1970); hence this process may be important for a very restricted class of objects (e.g. molecular cloud classifications $\mathrm{Cl}$ and $\mathrm{C} 2$, discussed previously).

(3) Dissociation of More Complex Molecules. It is possible that some of the molecular species are direct byproducts of more complex molecules. Carbon monoxide (CO), for example, can be formed by both vapor-phase reactions or by the photodistruction of formaldehyde (Glicker and Stief, 1971). So it is not unlikely that large accumulations of some of the structurally simpler interstellar molecules mark the 'graveyard' of more complex species. But the dissociation approach to molecular formation tends to beg the question of how the more complex parent molecules are formed.

(4) Reactions Involving Grains. Until recently, there was a tendency to downgrade the role of grains in the molecular formation process. Perhaps we are at a turning point in astronomical thinking regarding the interrelationship between dust grains and molecular formation when we see the papers presented at this meeting which quantitatively employ the grains as a source of interstellar molecules (Greenberg, 1972, 1973; Salpeter and Watson, 1973; Greenberg and Yencha, 1973). Introducing grains to explain the astrochemistry appears to be the most promising approach for satisfying the conditions beginning to emergy from the observations. Observationally we see strong (but not yet conclusive) suggestions of preferred reaction routes for molecular formation. Some of these are: 
(a) Structural correlations - Some or all of these may be due to observational selection effects but it is noteworthy that numerous species of the form $\mathrm{HCO} X$ have been observed where $X=\mathrm{H}$ (formaldehyde), $\mathrm{OH}$ (formic acid), $\mathrm{NH}_{2}$ (formamide), or $\mathrm{CH}_{3}$ (acetaldehyde). Another structural correlation based on formaldehyde is of the form $\mathrm{H}_{2} \mathrm{C} Y$ where $Y=\mathrm{O}$ (formaldehyde), $\mathrm{S}$ (thioformaldehyde) or NH (methylenimine). At first glance, these structural correlations appear to support vapor-phase formation reactions of the form $\mathrm{HCO}+X \rightarrow \mathrm{HCO} X$. However, such simple two-body reactions are not probable because radio observers have failed to detect the HCO radical.

(b) Molecular intermediates and unidentified molecules - molecular intermediates such as methylenimine $\left(\mathrm{CH}_{2} \mathrm{NH}\right)$ and thioformaldehyde $\left(\mathrm{H}_{2} \mathrm{CS}\right)$ are quickly destroyed by wall collisions in terrestrial laboratories. Unidentified compounds such as $X$-ogen and 'HNC' may be molecules with such short terrestrial lifespans that we may eventually classify them as 'nonterrestrial' molecules. Both classes of short-lived molecules suggest that low temperature matrix-phase chemistry (where a grain serves as the matrix) is important to interstellar formation.

(c) Negative results - not many negative observations are yet regarded as truly definitive, but it is worth mentioning that the failure to find simple compounds containing a nitrogen-oxygen bond (e.g. NO) lends substance to the idea that there are strongly preferred formation reactions for interstellar molecules. This perhaps would be surprising if vapor-phase reactions were the only allowed formation process.

\section{Conclusion}

In this review, I've attempted to relate observed molecular species and their abundances to the idea of cloud evolution. Before we can classify clouds and build meaningful models, we have to know more about the formation and excitation of interstellar molecules. The evidence is growing that interstellar dust grains are central to the understanding of molecular formation and hence play an important role in understanding cloud evolution. In turn, the molecules probably are our best tool for the quantitative analysis of the grain composition.

\section{Acknowledgement}

I wish to acknowledge partial support from National Science Foundation Grant GP-34200.

\section{References}

Buhl, D.: 1971, Nature 234, 332.

Buhl, D. and Snyder, L. E.: 1971, Tech. Rev. 73, 54.

Davies, R. D. and Matthews, H. E.: 1972, Monthly Notices Roy Astron. Soc. 156, 253.

Glicker, S. and Stief, L. J.: 1971, J. Chem. Phys. 54, 2852.

Greenberg, L. T.: 1973, this volume, p. 413.

Greenberg, J. M.: 1972, in M. A. Gordon and L. E. Snyder (eds.), Molecules in the Galactic Environment, Wiley Interscience, New York. 
Greenberg, J. M. and Yencha, A. J.: 1973, this volume, p. 369.

Heiles, C.: 1971, Ann. Rev. Astron. Astrophys. 9, 293.

Julienne, P. S., Krauss, M., and Donn, B.: 1971, Astrophys. J. 170, 65.

McNally, D.: 1968, Adv. Astron. Astrophys. 6, 173.

Rank, D. M., Townes, C. H., and Welch, W. J.: 1971, Science 174, 1083.

Salpeter, E. E. and Watson, W. D.: 1973, this volume, p. 363.

Snyder, L. E.: 1972, in D. A. Ramsay (ed.) MTP International Review of Science, Physical Chemistry Series One, Volume 3: Spectroscopy, Butterworth \& Co. Ltd., London, p. 193.

Snyder, L. E. and Buhl, D.: 1970, Sky Telesc. 40, 267.

Solomon, P. M. and Klemperer, W.: 1972, Astrophys. J. 178, 389.

Stecher, T. P. and Williams, D. A.: 1973, to be published.

Thaddeus, P., Kutner, M. L., Penzias, A. A., Wilson, R. W., and Jefferts, K. B.: 1972, Astrophys. J. Letters 176, L73.

Turner, B. E.: 1970, Publ. Astron. Soc. Pacific 82, 996. 